\title{
Analítica de la percepción de docentes y estudiantes sobre el uso de metodologías de enseñanza tradicionales e innovadoras en educación superior
}

\section{Analysis of the perception of teachers and students on the use of traditional and innovative teaching methodologies in higher education}

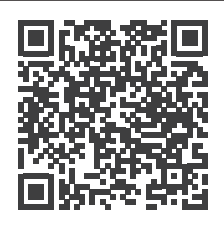

\section{Palabras clave:}

educación económica y financiera, educación, estrategias de aprendizaje, metodologías, tecnologías de la información y de la comunicación.

Artículo de investigación:

Fecha de recepción: $18 / 08 / 2020$

Fecha de aceptación: 05/02/2021

Esta publicación se encuentra bajo licencia:

Creative Commons Reconocimiento-

NoComercial-

SinObraDerivada 4.0 Internacional

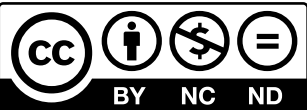

María Eugenia Morales-Sierra ${ }^{1}$; Hans Molano-Cardeño²; Daniel Cardona-Valencia3; Daniela Delgado-Cadavid ${ }^{4}$

\section{Resumen}

La valoración de las variables cualitativas en estrategias de enseñanza-aprendizaje es un factor clave en el desarrollo de las estrategias educativas que fortalezcan el aprendizaje, apoyado en las TIC. Este fortalecimiento incluye aplicar metodologías innovadoras y actividades didácticas, que logren de una mejor manera la transmisión y recepción del conocimiento. El objetivo de este trabajo es evaluar un modelo de aceptación tecnológica (TAM) a partir de un cuestionario dirigido a docentes y estudiantes de los campos disciplinares económico y financieros de instituciones públicas y privadas de educación superior en Medellín, Colombia, para determinar el nivel de incidencia de las variables: actitud, utilidad percibida, facilidad de uso, intención y confianza,

1 Ingeniera financiera y de negocios. Magíster en Administración Financiera, Politécnico Gran Colombiano. memorales@poligran.edu.co ORCID: 0000-00027702-0398

2 Ingeniero financiero y de negocios, Instituto Tecnológico Metropolitano. hanzzmolanoo8989@gmail.com ORCID: 0000-0001-5251-4466

3 Ingeniero industrial. Doctor en Desarrollo y Cooperación Internacional (c), Instituto Tecnológico Metropolitano. danielcardona@itm.edu.co ORCID: 00000001-8689-4399

4 Ingeniera financiera y de negocios, Instituto Tecnológico Metropolitano. danieladelgado236370@correo.itm.edu.co ORCID: 0000-0002-8183-427 


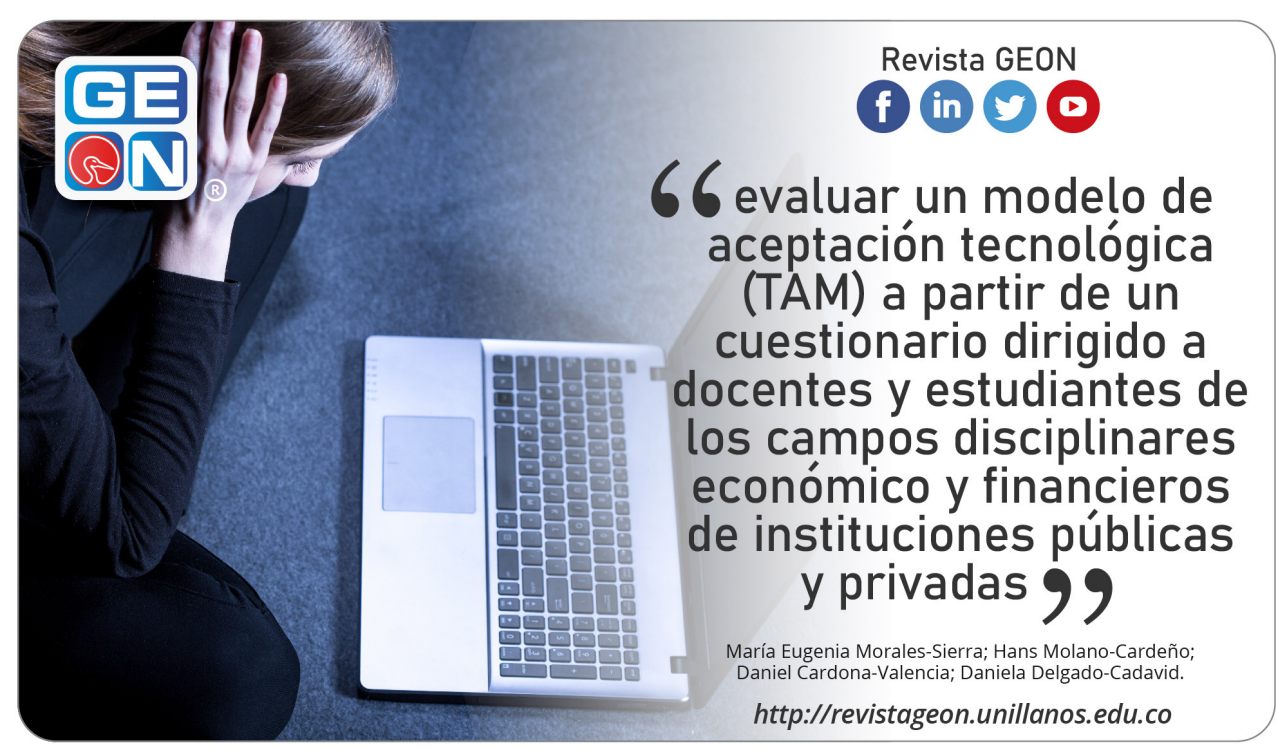

frente a las metodologías didácticas. Se presenta una investigación de tipo descriptivo, apoyada en técnicas y métodos de validación como la prueba de Bartlett, KMO, Alfa de Cronbach y la validación convergente y discriminante para los constructos del modelo. Como resultado se evidencia una asociación positiva entre las variables estudiadas y se valida la pertinencia de las metodologías para el grupo de estudio con alto grado de aceptación en estrategias como exámenes, talleres y juegos como prácticas académicas.

Se logra validar la pertinencia de las metodologías de enseñanza-aprendizaje tanto innovadoras como tradicionales para el grupo de estudio y se obtiene una alta aceptación; asimismo, se halla un alto grado de aceptación en el uso de exámenes, talleres y juegos, los cuales sugieren que en la actualidad

Cómo citar este artículo / Toreference this article:

Morales Sierra, M. E., MolanoCardeño, H., Cardona-Valencia, D., \& Delgado-Cadavid, D. (2021). Analítica de la percepción de docentes y estudiantes sobre el uso de metodologías de enseñanza tradicionales e innovadoras en educación superior. Revista GEON (Gestión, Organizaciones Y Negocios), 8(1), e-224. https://doi org/10.22579/23463910.224 se aplican efectivamente como herramientas de aprendizaje.

Palabras clave: educación económica y financiera, educación, estrategias de aprendizaje, metodologías, tecnologías de la información y de la comunicación.

Código JEL: I22 Finanzas educativas; Ayuda financiera, 1120 Educación 
Cómo citar este artículo / Toreference this article:

Morales Sierra, M. E., MolanoCardeño, H., Cardona-Valencia, D., \& Delgado-Cadavid, D. (2021) Analítica de la percepción de docentes y estudiantes sobre el uso de metodologías de enseñanza tradicionales e innovadoras en educación superior. Revista GEON (Gestión, Organizaciones Y Negocios), 8(1), e-224. https://doi. org/10.22579/23463910.224

\section{Abstract}

The valuation of qualitative variables in teaching-learning strategies is a key factor in the development of educational strategies that strengthen learning, supported by ICT. This strengthening includes the application of innovative methodologies and didactic activities to achieve a better transmission and reception of knowledge.

The objective of this research is to evaluate a technological acceptance model (TAM) based on a questionnaire addressed to teachers and students of a public institution of higher education in MedeIlin, Colombia, to determine the level of incidence of the variables: attitude, perceived utility, ease of use, intention and confidence, in comparison to didactic methodologies. We present a descriptive research supported by validation techniques and methods such as the Bartlett test, KMO, Cronbach's Alpha and the convergent and discriminant validation for the constructs of the model. As a result, the degree of favorability in the perceived relevance for both traditional and innovative trends is presented, evidencing a positive association between the construct variables studied without detriment or harm in the existence of putting into action an innovative tool within the classroom.

Keywords: Economic and financial education; Education; Information and communication technologies; Learning strategies; Methodologies; Validity; Reliability.

\section{Introducción}

Los establecimientos académicos son herramientas que promueven las didácticas en las clases, para potencializar las habilidades estudiantiles a fin de mejorar la calidad en la formación (Turbay, 2000). Pero ante la evolución de la tecnología y la educación, se debe cuestionar la eficacia de los métodos y las estrategias ejercidas en la formación de profesionales.

No obstante, la deserción en la academia está presente al igual que los factores que motivan este comportamiento, como la falta de recursos financieros, capital cultural y académi- 
co y la formación u orientación profesional por parte de las instituciones y profesionales (Ministerio de Educación Nacional, 2009).

De acuerdo con Vélez (2011), la educación es para todos independientemente de la etnia cultural, tal y como lo dicta la Constitución política de Colombia, y afirma que las metodologías de formación no son las más adecuadas para una educación óptima. Además, debido a la falta de estímulo en las TIC, se presentan dificultades en los procesos integrales para el desarrollo y una buena inclusión de la educación económica y financiera (Valencia, Giraldo, Valencia, Palacios \& Piedrahita, 2018). De otra manera, las estrategias de aprendizaje deberían orientarse a que el estudiante desarrolle un criterio propio, mediante las metodologías activas que promuevan este nuevo enfoque a través de la lúdica y los juegos, estimulando las comunicaciones interpersonales, la participación, la motivación y autonomía. De igual manera, hay estudios que evidencian el interés en las clases cuando los docentes utilizan prácticas sofisticadas para el aprendizaje (MINTIC, 2016).

Según el Ministerio de Educación Nacional (2006), es necesario que el sector educativo evolucione en calidad, proponiendo nuevos caminos acordes con el desarrollo socioeducativo. No obstante, pensando en el mejoramiento continuo, las herramientas contemporáneas se han acomodado al sistema económico de las naciones, y se ha esperado que las personas se adapten a los cambios globales (Valencia et al., 2018).
Pero la adopción de estos instrumentos sofisticados, que potencializan las estrategias de formación, ha tenido un acercamiento carente de información en los factores que definen el grado de aceptación y uso de las metodologías didácticas en la educación. De igual manera, la falta de comprensión sobre el potencial que podría desencadenar una buena utilización de las TIC. De acuerdo con esto: ¿es posible determinar la pertinencia de estrategias activas de aprendizaje en temas educativos financieros, administrativos y de gestión en instituciones de educación superior en la ciudad?

\section{Contexto teórico}

La educación permite estimular el crecimiento integral del individuo. Como modelo de formación técnica, fortalece el desarrollo de la capacidad intelectual mediante conocimientos teóricos y experimentales, que se pueden adaptar de manera constante y eficaz (Delors, 1996). Sin embargo, los medios convencionales que atienden la demanda formativa son poco viables (García, 1999); el siglo XXI presenta mayor exigencia en la educación, dado que los estudiantes requieren mayores conocimientos teóricos y técnicos adaptados a las nuevas necesidades de la sociedad del conocimiento (Delors, 1996).

Según la UNESCO (1995), el sistema educativo puede desempeñar un papel estratégico superando las restricciones que van desde el ámbito institucional; así mismo, debe mejorar la organización y la gestión de los procesos de tal forma que se convier- 
ta en una herramienta de transformación productiva con equidad.

Una estructura educativa con bases firmes de la formación fomenta el crecimiento de los ingresos, disminuye el desempleo, mejora la salud y erradica la pobreza en una nación, impulsando la economía y estimulando la innovación (Grupo Banco Mundial, 2018). En este sentido, desde el consumo de productos y servicios hasta el desarrollo de aptitudes y habilidades, afianzan una formación financiera óptima (García, Grifoni, López, \& Mejía, 2013). No obstante, con el objeto de fortalecer y crear nuevas estrategias en la educación financiera, debe existir sinergia entre los recursos públicos y propios de las instituciones de educación superior (IES) (Pérez \& Jara, 2006). Lo anterior, con el propósito de combatir el analfabetismo financiero y permitir la toma de mejores decisiones en las finanzas personales (Informe Español, 2012).

Por su parte, un estudio realizado en Francia buscó determinar la relación entre la planeación y la educación financiera; se encontró que las personas que tienen conceptos financieros claros se comprometen más con sus planes económicos (Arrondel, Debbich \& Savignac, 2013). Asimismo, en Rusia se analizó la incidencia del crecimiento de las deudas de los hogares con la educación financiera y las decisiones tomadas por las personas; se encontró que existe un vínculo entre los mercados y la educación financiera (Klapper, Lusardi \& Panos, 2013).

El uso de las TIC se ha convertido en apoyo en los procesos de formación académica, de modo que sus agentes afinen los conceptos y términos. En este sentido, observamos cómo los alumnos constantemente son influenciados por los dispositivos electrónicos, celulares, televisión digital, videojuegos y la utilización diaria del internet (Hermosa Del vasto, 2014); cabe mencionar que las metodologías activas se apoyan en las TIC (Gazca-Herrera, Sánchez-Hernández, Culebro-Castillo \& Zabala-Arriola, 2019).

La UNESCO publicó The ICT Competency Framework for Teachers (Marco de Competencia TIC para Maestros, en español), el cual tiene como objetivo ayudar a los países a desarrollar políticas y estándares nacionales de competencia TIC para docentes. También busca resaltar que no es suficiente que los docentes posean competencias en TIC y logren transmitírselas a sus estudiantes, de forma adecuada, instándolos a desarrollar verdaderas redes colaborativas que permitan resolver problemáticas de forma más eficientes (UNESCO, 2020).

Por su parte, la CEPAL, en el Plan de Acción Regional sobre la Sociedad de la Información en América Latina y el Caribe (eLAC2010), contempla como primera prioridad en América Latina y el Caribe la incorporación de las TIC en la educación, especialmente como una contribución a los procesos de enseñanza-aprendizaje, definición que se acoge por considerar la educación como un factor relevante en la transformación social que permite el logro de la equidad (CEPAL, 2010).

Los juegos serios han ido abarcando el mercado con su industria innovadora y afinando técnicas, modelos 
y paradigmas, para potencializar el aprendizaje en la educación (Desarrollo Español de Vídeo Juegos, 2017). Incluso investigaciones afirman la relación existente entre el uso de videojuegos y el aprendizaje (Juca, García \& Burgo, 2017). Lo anterior indica que estar expuestos a nuevos desafíos, obtener logros y ser recompensado genera dopamina en el cerebro (Zichermann \& Cunningham, 2011).

En cuanto a los juegos serios, estos son juegos tecnológicos diseñados con un propósito más allá del mero entretenimiento; es decir, pensados y creados con fines educativos e informativos, por ejemplo, simuladores o juegos para crear conciencia (Dicheva et al., 2015). Este tipo de juegos sitúa al jugador o aprendiz en un contexto muy particular con el objetivo de desarrollar un conocimiento o habilidad específica. Por esta razón, es difícil incorporar un juego serio a una situación de aprendizaje diferente para la que fue creado.

La educación puede ser más eficiente si se logran integrar las diferentes estrategias de enseñanza-aprendizaje en los procesos formativos, que generen en los estudiantes motivación, estimulen la reflexión y la participación y desarrollen la autonomía durante la etapa de formación académica (López Noguero, 2005). Es así como el uso de los juegos en los procesos formativos, a través de los diferentes enfoques y tendencias, se ha convertido en una herramienta didáctica de interés en el desarrollo del aprendizaje de los estudiantes de diferentes niveles educativos, ya que los juegos permiten elegir alternativas, tomar decisiones y desarrollar habilidades para construir su propio conocimiento (Kark, 2011).

Con base en este sustento teórico, la adopción de herramientas que han permitido impulsar estrategias para la formación académica en las instituciones ha tenido un acercamiento carente de información (Pineda, 2020) en los factores que definen el grado de aceptación y uso de las metodologías didácticas, como los juegos serios, la lúdica y en general las estrategias pedagógicas que permitan acercar a los estudiantes con el conocimiento, mejorando así la aprehensión de conocimientos. Además de la falta de comprensión sobre el potencial que podría desencadenar una buena utilización de las TIC (Abbad, Morris \& de Nahlik, 2009).

\section{Métodos y materiales}

Para analizar las metodologías didácticas y el uso de juegos serios a partir de variables relacionadas con la aceptación por parte de estudiantes y docentes, se propone la aplicación de un modelo de aceptación tecnológica (TAM) en el cual se definen dimensiones o variables que de acuerdo con Yong Varela (2004) permiten identificar constructos de percepción social relacionados con la actitud de los actores, la utilidad percibida, la facilidad de uso, la intención y la confianza que les da dicha aplicación o cambio en una sociedad, estructura u organización.

Este instrumento propone la creación de una encuesta estructurada en escala Likert como lo proponen Alarcón y Araneda (2007); en la cual se identi- 
fiquen aspectos relevantes que puedan dar una idea sobre la percepción de los usuarios con relación a alguna novedad, particularmente relacionada con tecnología.

Luego del diseño del instrumento, se construyó una big data a través de una recolección de datos de una población total encuestada de 118 docentes, pertenecientes a una población total de 168 profesores, y de 407 estudiantes, de una población de 6000 alumnos, de diferentes instituciones académicas de carácter público y privadas de la ciudad de Medellín. El margen de error fue del $5 \%$ y con confianza del $95 \%$, con validación del contenido por un panel de expertos y la evaluación de la confiabilidad y validez del instrumento, que buscaba el nivel de incidencia de las metodologías de enseñanza-aprendizaje en la muestra mediante el programa estadístico SPSS, con el cual se realizaron las pruebas de esfericidad de Bartlett y Kaiser Mayer Olkin (KMO) (Carmona, 2014), validez convergente y discriminante (Lévy \& Varela, 2006) y análisis de fiabilidad a partir del alfa de Cronbach (González, 2008).

\section{Resultados}

A continuación, se muestran los resultados obtenidos luego de realizada la encuesta a los estudiantes y docentes.

Para medir el nivel de fiabilidad y validez de la incidencia de las variables latentes en el estudio, para Docentes se determinó:

- Alfa de Cronbach: los índices de confiabilidad están todos por en- cima de 0,80, lo que demuestra consistencia interna en cada constructo del modelo TAM cumpliendo con lo recomendado, entre 0,70 y 0,90 (González, 2008), como se muestra en la Tabla 1.

Tabla 1. Alfa e Cronbach para los constructos de docentes

\begin{tabular}{lc}
\multicolumn{1}{c|}{ Factor } & Alfa de Cronbach \\
\hline Actitud & 0,816 \\
\hline Utilidad percibida & 0,807 \\
\hline Facilidad de uso & 0,830 \\
\hline Confianza & 0,880 \\
\hline Intención de uso & 0,911 \\
\hline
\end{tabular}

Fuente: elaboración propia. Extracción de datos del software SPSS.

- KMO y Bartlett: al estimar el KMO para los constructos del TAM en la encuesta, se evidencia que hay cumplimiento en su criterio, por su ubicación entre 0,790 y 0,828. Esto quiere decir que se puede continuar con el análisis factorial. Además, cumplen la prueba de esfericidad de Bartlett, ya que los valores de significancia tienden a cero (Tabla 2).

- Validez convergente: el estudio en su validación convergente para los factores incluyentes al TAM para docentes indica, como se muestra en la

- Tabla 3, que las cargas factoriales cumplen con el primer criterio de validación convergente, a excepción de un reactivo, que tuvo una carga factorial de 0,364 en el constructo "Utilidad percibida" (E1_UP4). De acuerdo con lo anterior, este no aporta buena significancia al grado de asociación con 
la utilidad percibida, por lo que se elimina la afirmación que relaciona un buen rendimiento académico cuando se aplican los juegos serios en clase. El segundo criterio, el promedio de las cargas factoria- les estandarizadas, debe dar por encima de 0,7 y absolutamente todos los constructos lo cumplen, excediendo un promedio de carga factorial en promedio entre 0,721 y 0,828 .

Tabla 2. KMO y Bartlett para los constructos de profesores

\begin{tabular}{lc|c|c}
\multicolumn{1}{c|}{ Factor } & Valor KMO & Valor Bartlett & Cumple criterios \\
\hline Actitud & 0,735 & 0,00 & Sí \\
\hline Utilidad percibida & 0,795 & 0,00 & Sí \\
\hline Facilidad de uso & 0,790 & 0,00 & Sí \\
\hline Confianza & 0,811 & 0,00 & Sí \\
\hline Intención de uso & 0,828 & 0,00 & \\
\hline
\end{tabular}

Fuente: elaboración propia. Extracción de datos del software SPSS.

Tabla 3. Análisis de validez convergente para los ítems de profesores

\begin{tabular}{|c|c|c|c|}
\hline Constructo & Ítem & $\begin{array}{l}\text { Cargas factoriales } \\
\text { estandarizadas }\end{array}$ & $\begin{array}{c}\text { Promedio de cargas } \\
\text { factoriales estandarizadas }\end{array}$ \\
\hline \multirow{5}{*}{ Actitud } & E1_ACT1 & 0,506 & \multirow{5}{*}{0,731} \\
\hline & E1_ACT2 & 0,677 & \\
\hline & E1_ACT3 & 0,844 & \\
\hline & E1_ACT4 & 0,881 & \\
\hline & E1_ACT5 & 0,747 & \\
\hline \multirow{5}{*}{$\begin{array}{l}\text { Utilidad } \\
\text { percibida }\end{array}$} & E1_UP1 & 0,772 & \multirow{5}{*}{0,721} \\
\hline & E1_UP2 & 0,754 & \\
\hline & E1_UP3 & 0,861 & \\
\hline & E1_UP4 & 0,364 & \\
\hline & E1_UP5 & 0,854 & \\
\hline \multirow{5}{*}{$\begin{array}{l}\text { Facilidad } \\
\text { de uso }\end{array}$} & E1_FU1 & 0,553 & \multirow{5}{*}{0,752} \\
\hline & E1_FU2 & 0,538 & \\
\hline & E1_FU3 & 0,881 & \\
\hline & E1_FU4 & 0,892 & \\
\hline & E1_FU5 & 0,895 & \\
\hline \multirow{5}{*}{ Confianza } & E1_CON1 & 0,814 & \multirow{5}{*}{0,783} \\
\hline & E1_CON2 & 0,788 & \\
\hline & E1_CON3 & 0,637 & \\
\hline & E1_CON4 & 0,882 & \\
\hline & E1_CON5 & 0,794 & \\
\hline \multirow{5}{*}{$\begin{array}{l}\text { Intención } \\
\text { de uso }\end{array}$} & E1_INT1 & 0,629 & \multirow{5}{*}{0,828} \\
\hline & E1_INT2 & 0,87 & \\
\hline & E1_INT3 & 0,845 & \\
\hline & E1_INT4 & 0,913 & \\
\hline & E1_INT5 & 0,885 & \\
\hline
\end{tabular}

Fuente: construcción propia a partir de software estadístico SPSS. Acrónimos en anexo 1. 
- Validez discriminante: evidencia la relación que tiene cada variable latente del TAM. Se utiliza como referencia la validación de la prueba con el intervalo de confianza, para cada uno de los casos. Al no contener el 1 en el intervalo de confianza, los conceptos entre constructos se separan entre ellos, lo que determina que hay validez discriminante. Según Manzano y Jiménez (2017)comprobación de las propiedades de normalidad, homocedasticidad, linealidad e independiencia de las observaciones, la asociación entre variables es una estimación puntual con un error estándar; entonces, se sugiere construir un intervalo de confianza alrededor de dicha estimación).

- Tabla 4).

De igual manera, para los estudiantes se calculó el nivel de fiabilidad y validez de la incidencia de las variables latentes en el estudio, tal y como se muestra a continuación.

- Alfa de Cronbach: de acuerdo con la Tabla 5, se evidencia que las variables latentes cumplen con el criterio y se menciona que los cinco constructos tienen consistencia interna y el instrumento dirigido a estudiantes es fiable.

Tabla 4. Análisis de validez discriminante para los ítems de profesores

\begin{tabular}{l|c|c}
\multicolumn{4}{c}{ Correlación de Spearman } & \\
& Constructos cruzados & Intervalo de confianza del 95\% \\
\cline { 2 - 3 } Actitud $\longrightarrow$ Utilidad percibida & Inferior & Superior \\
\hline Actitud $\longrightarrow$ Facilidad de uso & 0,530 & 0,767 \\
\hline Actitud $\longrightarrow$ Confianza & 0,558 & 0,796 \\
\hline Actitud $\longrightarrow$ ntención & 0,575 & 0,815 \\
\hline Utilidad percibida $\longrightarrow$ Actitud & 0,514 & 0,769 \\
\hline Utilidad percibida $\longrightarrow$ Facilidad de uso & 0,530 & 0,760 \\
\hline Utilidad percibida $\longrightarrow$ Confianza & 0,469 & 0,725 \\
\hline Utilidad percibida $\longrightarrow$ Intención & 0,547 & 0,777 \\
\hline Facilidad de uso $\longrightarrow$ Actitud & 0,526 & 0,791 \\
\hline Facilidad de uso $\longrightarrow$ Utilidad percibida & 0,566 & 0,789 \\
\hline Facilidad de uso $\longrightarrow$ Confianza & 0,466 & 0,731 \\
\hline Facilidad de uso $\longrightarrow$ Intención & 0,517 & 0,767 \\
\hline Confianza $\longrightarrow$ Actitud & 0,587 & 0,835 \\
\hline Confianza $\longrightarrow$ Utilidad percibida & 0,572 & 0,804 \\
\hline Confianza $\longrightarrow$ Facilidad de uso & 0,546 & 0,777 \\
\hline Confianza $\longrightarrow$ Intención & 0,517 & 0,778 \\
\hline Intención $\longrightarrow$ Actitud & 0,687 & 0,869 \\
\hline Intención $\longrightarrow$ Utilidad percibida & 0,515 & 0,767 \\
\hline Intención $\longrightarrow$ Facilidad de uso & 0,537 & 0,791 \\
\hline Intención $\longrightarrow$ Confianza & 0,591 & 0,834 \\
\hline Funte: & 0,686 & 0,877
\end{tabular}

Fuente: construcción propia a partir del software estadístico SPSS. 
Tabla 5. Alfa de Cronbach para los constructos de estudiantes

\begin{tabular}{l|l}
\multicolumn{1}{c|}{ Factor } & \multicolumn{1}{c}{ Alfa de Cronbach } \\
\hline Actitud & 0,844 \\
\hline Utilidad percibida & 0,914 \\
\hline Facilidad de uso & 0,767 \\
\hline Confianza & 0,899 \\
\hline Intención de uso & 0,910 \\
\hline
\end{tabular}

Fuente: elaboración propia. Extracción de datos del software SPSS.

- KMO y Balrtlett: en los coeficientes KMO para los estudiantes, se determinó que las variables latentes cumplen con coeficientes moderados, lo que afirma que se puede realizar un análisis factorial para los constructos. En la prueba de esfericidad de Bartlett, el valor de $p$ es menor de 0,05 para todos los casos ajustados a los criterios del estudio (

- Tabla 6).

- Validez convergente: a partir de la información de los estudiantes, las cargas factoriales de los construc- tos observados en la Tabla 7 para el primer criterio de validación convergente se cumplen. A excepción de 3 reactivos con cargas factoriales de 0,437, 0,321 y 0,262, 1 en la actitud de los estudiantes y 2 en su facilidad de uso, de acuerdo con lo mencionado, estos ítems se eliminan; estos cuestionan los juegos en clase con el aprendizaje adquirido durante esta, su rapidez y la aplicación de juegos con poco esfuerzo y la dificultad de aquellos en los cursos porque requieren mucho tiempo de desarrollo. No son ítems que aporten buena significancia por parte de los estudiantes a la "actitud" y" facilidad de uso", entonces, no miden lo que se supone que deberían medir, es decir, sus respectivas variables latentes.

- Validez discriminante: de acuerdo con la tabla 8, los constructos del TAM direccionados a estudiantes muestran validez discriminante, una vez que se tienen intervalos de confianza con valores menores que uno.

Tabla 6. KMO y Bartlett para los constructos de estudiantes

\begin{tabular}{lc|c|c}
\multicolumn{1}{c|}{ Factor } & Valor KMO & Valor Bartlett & Cumple criterios \\
\hline Actitud & 0,781 & 0,000 & Sí \\
\hline Utilidad percibida & 0,875 & 0,000 & Sí \\
\hline Facilidad de uso & 0,755 & 0,000 & Sí \\
\hline Confianza & 0,866 & 0,000 & Sí \\
\hline Intención de uso & 0,866 & 0,000 & Sí \\
\hline
\end{tabular}

Fuente: elaboración propia. Extracción de datos del software SPSS. 
Tabla 7. Análisis de validez convergente para los ítems de estudiantes

\begin{tabular}{|c|c|c|c|}
\hline Constructo & Ítem & $\begin{array}{c}\text { Cargas factoriales } \\
\text { estandarizadas }\end{array}$ & $\begin{array}{c}\text { Promedio de cargas factoriales } \\
\text { estandarizadas }\end{array}$ \\
\hline \multirow{5}{*}{ Actitud } & E1_ACT1 & 0,437 & \multirow{5}{*}{0,725} \\
\hline & E1_ACT2 & 0,540 & \\
\hline & E1_ACT3 & 0,892 & \\
\hline & E1_ACT4 & 0,876 & \\
\hline & E1_ACT5 & 0,879 & \\
\hline \multirow{5}{*}{$\begin{array}{l}\text { Utilidad } \\
\text { percibida }\end{array}$} & E1_UP1 & 0,713 & \multirow{5}{*}{0,823} \\
\hline & E1_UP2 & 0,843 & \\
\hline & E1_UP3 & 0,868 & \\
\hline & E1_UP4 & 0,832 & \\
\hline & E1_UP5 & 0,857 & \\
\hline \multirow{5}{*}{ Facilidad de uso } & E1_FU1 & 0,321 & \multirow{5}{*}{0,650} \\
\hline & E1_FU2 & 0,262 & \\
\hline & E1_FU3 & 0,880 & \\
\hline & E1_FU4 & 0,900 & \\
\hline & E1_FU5 & 0,887 & \\
\hline \multirow{5}{*}{ Confianza } & E1_CON1 & 0,773 & \multirow{5}{*}{0,807} \\
\hline & E1_CON2 & 0,846 & \\
\hline & E1_CON3 & 0,847 & \\
\hline & E1_CON4 & 0,716 & \\
\hline & E1_CON5 & 0,852 & \\
\hline \multirow{5}{*}{ Intención de uso } & E1_INT1 & 0,602 & \multirow{5}{*}{0,819} \\
\hline & E1_INT2 & 0,828 & \\
\hline & E1_INT3 & 0,890 & \\
\hline & E1_INT4 & 0,881 & \\
\hline & E1_INT5 & 0,893 & \\
\hline
\end{tabular}

Fuente: elaboración propia. Extracción de datos del software SPSS. Acrónimos en anexo 1.

Tabla 8. Análisis de la validez discriminante para los ítems de estudiantes

\begin{tabular}{l|c|c}
\multicolumn{3}{c}{ Correlación de Spearman } \\
\multicolumn{1}{c|}{ Constructos cruzados } & \multicolumn{2}{c}{ Intervalo de confianza del 95\% } \\
\cline { 2 - 3 } & Inferior & \multicolumn{1}{c}{ Superior } \\
Actitud $\longrightarrow$ Utilidad percibida & 0,606 & 0,738 \\
\hline Actitud $\longrightarrow$ Facilidad de uso & 0,670 & 0,783 \\
\hline Actitud $\longrightarrow$ Confianza & 0,570 & 0,720 \\
\hline Actitud $\longrightarrow$ Intención & 0,604 & 0,737 \\
\hline Utilidad Percibida $\longrightarrow$ Actitud & 0,611 & 0,739 \\
\hline Utilidad Percibida $\longrightarrow$ Facilidad de uso & 0,637 & 0,758 \\
\hline Utilidad Percibida $\longrightarrow$ Confianza & 0,646 & 0,779 \\
\hline Utilidad Percibida $\longrightarrow$ Intención & 0,680 & 0,794
\end{tabular}




\begin{tabular}{l|c|c}
\multicolumn{3}{c}{ Correlación de Spearman } \\
& & \\
& Constructos cruzados & Intervalo de confianza del 95\% \\
\cline { 2 - 3 } & Inferior & Superior \\
\hline Facilidad de uso $\longrightarrow$ Actitud & 0,675 & 0,787 \\
\hline Facilidad de uso $\longrightarrow$ Utilidad percibida & 0,627 & 0,755 \\
\hline Facilidad de uso $\longrightarrow$ Confianza & 0,627 & 0,757 \\
\hline Facilidad de uso $\longrightarrow$ Intención & 0,690 & 0,812 \\
\hline Confianza $\longrightarrow$ Actitud & 0,566 & 0,716 \\
\hline Confianza $\longrightarrow$ Utilidad percibida & 0,648 & 0,779 \\
\hline Confianza $\longrightarrow$ Facilidad de uso & 0,631 & 0,753 \\
\hline Confianza $\longrightarrow$ Intención & 0,646 & 0,778 \\
\hline Intención $\longrightarrow$ Actitud & 0,604 & 0,738 \\
\hline Intención $\longrightarrow$ Utilidad percibida & 0,672 & 0,800 \\
\hline Intención $\longrightarrow$ Facilidad de uso & 0,693 & 0,813 \\
\hline Intención $\longrightarrow$ Confianza & 0,654 & 0,779 \\
\hline
\end{tabular}

Fuente: elaboración propia. Extracción de datos del software SPSS.

\section{Análisis sobre la pertinencia y favorabilidad de los datos derivados de las metodologías tradicionales e innovadoras, para agentes de formación}

\section{Docentes}

En el instrumento se toman en cuenta las metodologías tradicionales de enseñanza y las metodologías activas o también llamadas innovadoras; de acuerdo con esto, cada profesor encuestado se caracteriza según la tendencia tradicional o innovadora que aplique en sus clases.

En consecuencia, se evidencia que 73 docentes, equivalentes al 62\% de estos, usan herramientas innovadoras en sus aulas de clase, mientras que los restantes 45, es decir, el 38\%, realizan las clases con metodologías tradicionales. Lo anterior refiere que los docentes toman gran partida en la elaboración de la clase aprovechando herramientas lúdicas útiles para cautivar a los estudiantes en el proceso formativo.

El instrumento se diseñó utilizando la metodología TAM, con la cual se realizó una modelación de ecuaciones estructurales con mínimos cuadrados parciales (PLS-SEM, por sus siglas en inglés) (Yong Varela, 2004).

El cuestionario se estructuró a partir de preguntas cerradas, dicotómicas y multicotómicas, con el fin de obtener información referente al uso de los juegos serios como estrategia de enseñanza y, adicionalmente, conocer los estados internos del individuo con respecto a la actitud, la utilidad percibida, la facilidad de uso, la confianza 
y la intención de uso de los juegos serios. Se evaluaron cinco categorías clasificadas por: actitud, utilidad percibida, facilidad de uso, intención y confianza como estrategia didáctica e integradora en procesos presenciales y de e-learning.

De acuerdo con la información recolectada en la encuesta de los docentes, se logran caracterizar dos bases de datos, según las tendencias tradicionales e innovadoras de los docentes en el aula de clase. Se trabajan las bases de datos en dos tablas, y se hace un gráfico descriptivo de la información; esto con el propósito de resumir los datos y ver la asociación y la pertinencia entre estos dos grupos y las estrategias de enseñanza.

En la Figura 1, los profesores que se caracterizan por exclusivamente hacer uso de los métodos de enseñanza tradicionales no sienten detrimento ni perjuicio en poner en acción una herramienta innovadora dentro del aula de clase como los juegos serios; esto se evidencia por los altos niveles de favorabilidad frente al uso de estas herramientas en clase. También se valida la pertinencia de las metodologías innovadoras por los niveles favorables de los constructos evidenciados por el alto grado de aceptación de las tendencias tradicionales utilizadas por la docencia.

En la información de la Figura $\mathbf{2}$ se evidencia alta favorabilidad entre los componentes que inciden en los juegos serios aplicados para la enseñanza. Además, se valida la pertinencia de las metodologías innovadoras, como estudios de casos, gamificación, juegos de roles, lúdicas y experiencias, entre otras, debido a la aceptación del uso de los juegos serios por parte de la docencia, que acude a herramientas con base en las TIC. No obstante, también hay una alta aceptación de las tendencias tradicionales, como clases magistrales y lecturas desde un punto de vista más innovador en el desarrollo de los cursos.

Figura 1. Grado de favorabilidad de los juegos serios según la percepción de los docentes tradicionales. Fuente: elaboración propia con base en la información recolectada.

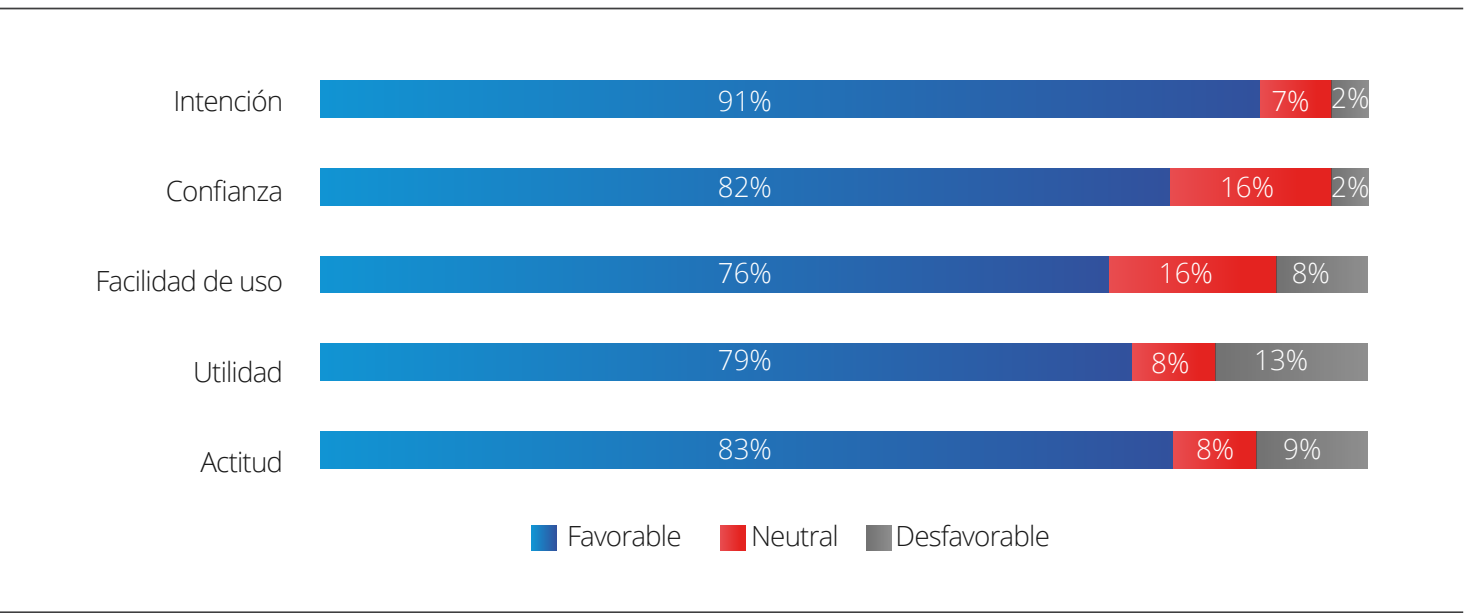


Figura 2. Grado de favorabilidad de los juegos serios según la percepción de los docentes innovadores. Fuente: elaboración propia, con base en la información recolectada.

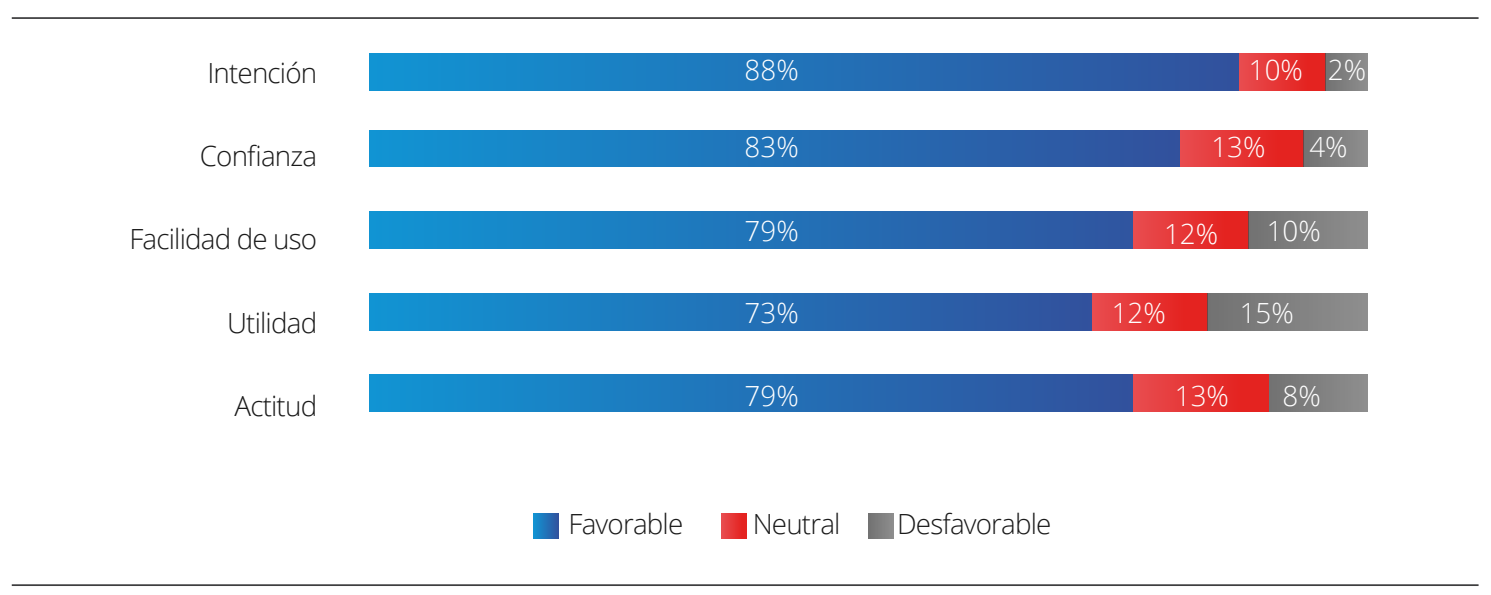

\section{Estudiantes}

Se evidencia que el $67 \%$ de los estudiantes evaluados, equivalente a 273 personas, han percibido metodologías tradicionales en las clases; es decir, aquellos métodos de aprendizaje toman gran partida en la elaboración de la clase con el objetivo de afianzar los conocimientos financieros. Asimismo, las actividades que los estudiantes reconocen con mayor frecuencia en sus clases son los exámenes, los talleres, las mesas redondas, los ensayos y las exposiciones. En una escala inferior, el 33\% restante, correspondiente a 134 personas, logra percibir metodologías de enseñanza que involucran herramientas sofisticadas.

Se pudo identificar tendencias tradicionales e innovadoras de metodologías de enseñanza, caracterizadas en dos bases de datos, esto de acuerdo con lo que perciben los estudiantes en las clases magistrales. En consecuencia, se construyen dos bases de datos que caracterizan estas tendencias, con el propósito de extraer los datos y ver la asociación y pertinencia entre estos dos grupos y las estrategias de enseñanza.

Figura 3 representa la percepción que tienen los estudiantes que reciben sus clases en un ambiente donde los docentes practican estrategias tradicionales para el afianzamiento del conocimiento en las finanzas, administración, gestión o cursos afines. Se muestra que aquellos estudiantes que reciben las clases con estas estrategias educativas tradicionales y su comportamiento con respecto a las categorías en los constructos del modelo TAM, enfocados en la aceptación de los juegos serios en clase, expresan alta favorabilidad hacia el uso de estos como herramienta en el proceso educativo; el resultado en cada una de las categorías es altamente positivo. En consecuencia, tienen un impacto de aceptación en las tendencias innovadoras, siendo estas estrategias tradicionales perti- 
nentes e importantes en el proceso y desarrollo de nuevas estrategias de innovación.

Para los estudiantes que afirman recibir clases con métodos innovadores y asociados a las TIC

Figura 4, su favorabilidad es altamente positiva. Incluso, en comparación con los estudiantes tradicionales, su variabilidad es mucho mayor, pues oscila entre 2 y 6 puntos porcentuales para todas las categorías del modelo TAM. De igual forma, así como este ítem tiene altos niveles de favorabilidad, también presenta niveles desfavorables, aunque estos son poco significativos en el modelo.
Tanto los estudiantes que perciben la aplicación de estrategias tradicionales en el aula de clase, como los estudiantes que perciben nuevas estrategias innovadoras y activas tienen alta favorabilidad en los constructos del modelo TAM, y están dispuestos a usar los juegos dentro del aula de clase o diferentes metodologías de aprendizaje.

En síntesis, debido a que las dos clases de estudiante evidencian altas posibilidades o favorabilidades en el modelo, hallamos en estas dos categorías una asociación en gran magnitud con tendencias positivas, que valida la pertinencia de las estrategias de enseñanza.

Figura 3. Grado de favorabilidad de los juegos serios según la percepción de los estudiantes tradicionales. Fuente: elaboración propia con base en la información recolectada.

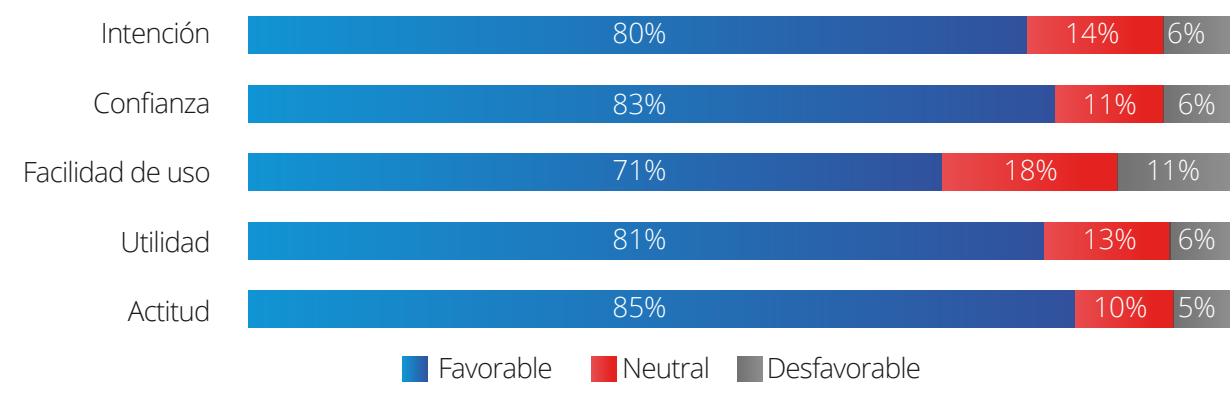

Figura 4. Grado de favorabilidad de los juegos serios según la percepción de los estudiantes innovadores. Fuente: elaboración propia con base en la información recolectada.

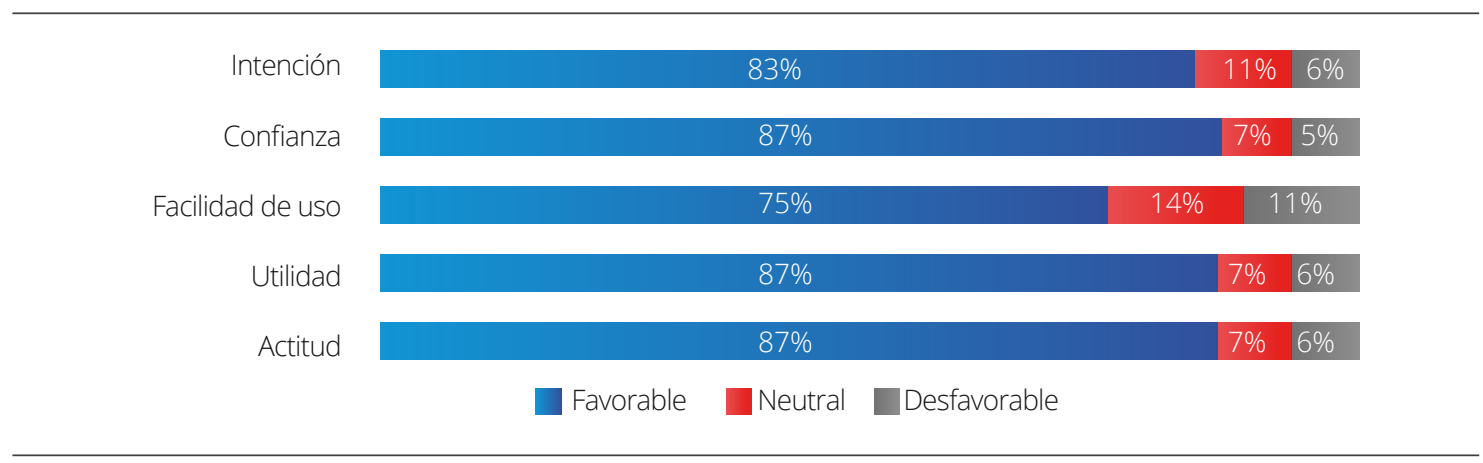




\section{Discusión}

El desarrollo de nuevas metodologías que permitan fortalecer el proceso enseñanza-aprendizaje continuará siendo un reto, en el que las comunidades educativas desempeñan un papel fundamental para propiciar los espacios que permitan minimizar las brechas entre las necesidades de la sociedad y la ampliación de la oferta académica como una contribución hacia la disminución de la desigualdad.

Las metodologías de enseñanza-aprendizaje, tanto innovadoras, a través de las herramientas pedagógicas, como los juegos, no solo permiten validar su pertinencia, también favorecen el escenario para crear comunidades colaborativas que motiven a docentes y estudiantes a buscar una mejor alternativa para la resolución de los problemas.

Se logra validar la pertinencia de las metodologías de enseñanza-aprendizaje innovadoras y de utilización de herramientas tecnológicas que permitan al estudiante vivir una experiencia simulada de su curso durante su desarrollo, con una alta aceptación, confianza e intención de uso por parte de los docentes frente a las metodologías tradicionales para el grupo de estudio; asimismo, se halla un alto grado de aceptación en el uso de exámenes, talleres y juegos, lo que sugiere que, en la actualidad, se aplican efectivamente como herramientas de aprendizaje que favorecen el proceso de enseñanza en las áreas económicas y financieras.

\section{Conclusiones}

Se logra validar la pertinencia de las metodologías de enseñanza-aprendizaje tanto innovadoras como tradicionales para el grupo de estudio, donde se obtuvo una alta aceptación. Asimismo, se encuentra un alto grado de aceptación en el uso de exámenes, talleres y juegos, lo que sugiere que, en la actualidad, se aplican efectivamente como herramientas de aprendizaje.

De acuerdo con este estudio, los profesores (categorizados como tradicionales e innovadores) muestran una alta favorabilidad por el uso de juegos serios para la enseñanza, con percepciones de utilidad, facilidad y confianza superiores al 79\% en favorabilidad.

Se destaca la utilidad percibida como el factor con menos favorabilidad para docentes, con una posición negativa sobre el uso de juegos serios del $14 \%$, en promedio. Por el contrario, para los estudiantes, la facilidad de desarrollo es el factor que mayor desfavorabilidad presenta, con un $11 \%$, en promedio.

Los estudiantes clasificados como innovadores (que ya han usado estrategias no tradicionales en sus clases) no desmejoran su percepción del aprendizaje basado en estrategias innovadoras después de su uso, en comparación con los estudiantes clasificados como tradicionales (que solamente han recibido clase magistral). Por el contrario, los docentes que ya usan o han usado las estrategias innovadoras muestran una disminución 
en la percepción de estas actividades, justificada principalmente en que representan más trabajo para el docentey en que no siempre tienen facilidad de uso en entornos tradicionales.

Los resultados estadísticos evidencian que no hay relación de causalidad entre las variables comparables entre alumnos y maestros, sin hallar detrimento ni perjuicio al poner en acción una herramienta innovadora, como lo es el "juego serio", dentro del aula. Hábilmente, tanto profesores como estudiantes muestran altos niveles de favorabilidad ante la intención, la confianza, la facilidad de uso, la utilidad y, sobre todo, la actitud frente al uso de juegos como herramienta innovadora de enseñanza en clase.

Se observa, además, que los estudiantes perciben que las actividades con metodología tradicional son las que se implementan con mayor frecuencia en las clases. No obstante, de acuerdo con esta evidencia, se comprueba también que el paradigma que se maneja socialmente respecto a las metodologías de enseñanza tradicionales tiene que ver con su aceptación como la mejor opción como modelo de enseñanza-aprendizaje. Sin embargo, no significa que las tendencias sean las más eficientes para potencializar los conocimientos teóricos y experimentales. Por otro lado, se evidencia que una pequeña parte de los encuestados presenta cierta resistencia por implementar nuevos métodos de enseñanza, de lo cual este estudio aclara que los estudiantes que reciben clases con actividades tradicionales son consecuentes con que las estrategias deban reestructurarse para potencializar los conocimientos. Esto se demuestra en los altos niveles de favorabilidad en el uso de nuevas estrategias de enseñanzas.

\section{Referencias}

Abbad, M. M., Morris, D. \& de Nahlik, C. (2009). Looking under the Bonnet: Factors Affecting Student Adoption of E-Learning Systems in Jordan. The International Review of Research in Open and Distributed Learning, 10(2). https:// bit.ly/3uuCMWh

Aldás, J. \& Uriel, E. (2017). Análisis multivariante aplicado con $R$ (2. ${ }^{\text {a }}$ ed.). Ediciones Paraninfo, SA.

Arrondel, L., Debbich, M. \& Savignac, F. (2013). Financial Literacy and Financial Planning in France. Numeracy, 6(2). https://bit.ly/2NVAkqY

Carmona, F. (2014). Un ejemplo de ACP paso a paso. Departamento de Estadística, 1-7. https://bit.ly/2NFOMDD

Comisión Económica para América Latina. (2010). Monitoreo del plan eLAC2010: avances y desafíos de la sociedad de la información en América Latina y el Caribe. Naciones Unidas. https://bit.ly/3bDHFUo

Delors, J. (1996). Los cuatro pilares de la educación. Informe a la UNESCO de la Comisión Internacional sobre la Educación para el siglo XXI. UNESCO.

Desarrollo Español de Videojuegos. (2017). Libro Blanco del Desarrollo Español de Videojuegos 2017. Fondo Europeo de Desarrollo Regional. https://bit.ly/3bzuKTn

Dicheva, D., Dichev C., Agre G. \& Angelova, G. (2015). Gamification in Edu- 
cation: A Systematic Mapping Study. Educational Technology \& Society, 18(3), 75-88. https://bit.ly/3uv6918

García-Aretio, L. (1999). Historia de la Educación a Distancia. Revista Iberoamericana de Educación a Distancia, 2(1), 11-40. https://bit.ly/37JnUJZ

García, N., Grifoni, A., López, J. C. \& Mejía, D. M. (2013). La educación financiera en América Latina y el Caribe. Situación actual y perspectivas. Serie Políticas Públicas y Transformación Productiva, 12. https://bit.ly/3kisicc

Gazca Herrera, L. A., Sánchez Hernández, G. L., Culebro Castillo, K., \& Zabala Arriola, O. (2019). Diagnóstico del uso de herramientas tecnológicas para la gestión y apoyo del liderazgo en las PYMES. Revista GEON (Gestión, Organizaciones Y Negocios), 6(1), 69-83. https://doi.org/10.22579/23463910.85

González-Ortega, Y. (2008). Instrumento cuidado de comportamiento profesional: validez y confiabilidad. Aquichan, 8(2), 170-82. https://bit.ly/2NFQgOd

Grupo Banco Mundial. (2018, noviembre). Aprender para hacer realidad la promesa de la educación. Banco Mundial. Consultado el 24 de febrero de 2021. https://bit.ly/3bRa5uj

Hermosa Del vasto, P. M. (2014). Influence of information and communication technologies (ICT) in the teaching-learning process: improving digital skills. Revista Científica General José María Córdova, 15(16), 121-132. https://bit. ly/3ssbais

Ministerio de Educación, Cultura y Deporte. (2012). PISA 2012 Competencia Financiera. Instituto Nacional de Evaluación en Educación. https://bit.ly/3dJfZAb
Juca-Maldonado, F., García-Saltos, M. B. \& Burgo-Bencomo, O. (2017). Los juegos serios y su influencia en el uso responsable de energía y cuidado del medio ambiente. Revista Universidad y Sociedad, 9(1), 129-136. https://bit. ly/3dWr9BN

Kark, R. (2011). Games managers play: Play as a form of leadership development. Academy of Management Learning and Education, 10(3), 507-527. https://bit.ly/2Mmjvvg

Klapper, L., Lusardi, A. \& Panos, G. A. (2013). Financial literacy and its consequences: Evidence from Russia during the financial crisis. Journal of Banking and Finance, 37(10), 3904-3923. https://bit.ly/3bBbViG

Lévy-Mangin, J. P. \& Varela-Mallou, J. (2006). Modelización con estructuras de covarianzas en ciencias sociales: temas esenciales, avanzados y aportaciones especiales. Netbiblo.

López-Noguero, F. (2005). Metodología participativa en Educación en la enseñanza universitaria. Ediciones de la U.

Ministerio de Educación Nacional. (2006). Retos e Indicadores del Sector Educativo. https://bit.ly/3aT5esU

Ministerio de Educación Nacional. (2009). Deserción estudiantil. https://bit.ly/3sqOgby

Ministerio de Tecnologías de la Información y las Comunicaciones. (2016). Las TIC siguen impactando positivamente la educación colombiana. Consultado el 12 de febrero de 2020. https://bit. Iy/2NAXT8!

Pérez Cano, M. \& Jara-Valls, M. del R. (2006). La gestión académica y financiera en la educación virtual. Apertura, 6(3), 37-49. https://bit.ly/3bzhM8d 
Pineda Henao, A. E. (2020). La comunicación organizacional en la gestión empresarial: retos y oportunidades en el escenario digital. Revista GEON (Gestión, Organizaciones Y Negocios), 7(1), 9-25. https://doi. org/10.22579/23463910.182

Reinoso-Alarcón, H. R. \& Araneda-Cea, B. G. A. (2007). Diseño y validación de un modelo de medición del clima organizacional basado en percepciones y expectativas. Revista Ingeniería Industrial, 6(1), 5. https://bit.ly/3dWny6L

Turbay-Restrepo, C. (2000). El derecho a la educación. Desde el marco de la protección integral de los derechos de la niñez y de la política educativa. Fundación Antonio Restrepo Barco. https://bit.ly/3aP7zFh

UNESCO. (1995). Innovación en la gestión educativa. Experiencias en Brasil, Chile y Venezuela. Oficina Regional de Educación de la UNESCO-OREALC. https:// bit.ly/2P9EOeh

UNESCO. (10 de agosto de 2020). Marco de competencias de los docentes en materia de TIC. UNESCO. https://bit.ly/2ZQBcjg

Valencia, D. C., Giraldo, C., Valencia, J., Palacios, L. \& Piedrahita, L. (2018). Relationship between ICT Use and Financial Education Levels in Latin America. International Journal of Innovation, Management and Technology, 9(4), 20169. https://bit.ly/3qOiyEC

Yong Varela, L. A. (2004). Modelo de aceptación tecnológica (TAM) para determinar los efectos de las dimensiones de cultura nacional en la aceptación de las TIC. Revista Internacional de Ciencias Sociales y Humanidades, 14(1), 131-71. https://bit.ly/37KxOWM
Zichermann, G. \& Cunningham, C. (2011). Gamification by Design: Implementing Game Mechanics in Web and Mobile Apps. O'Reilly.

\section{Anexo 1}

Acrónimos

E1_ACT1: Variable de actitud 1

E1_ACT2: Variable de actitud 2

E1_ACT3: Variable de actitud 3

E1_ACT4: Variable de actitud 4

E1_ACT5: Variable de actitud 5

E1_UP1: Variable de utilidad percibida 1

E1_UP2: Variable de utilidad percibida 2

E1_UP3: Variable de utilidad percibida 3

E1_UP4: Variable de utilidad percibida 4

19

E1_UP5: Variable de utilidad percibida 5

E1_FU1: Variable de facilidad de uso 1

E1_FU2: Variable de facilidad de uso 2

E1_FU3: Variable de facilidad de uso 3

E1_FU4: Variable de facilidad de uso 4

E1_FU5: Variable de facilidad de uso 5

E1_CON1: Variable de confianza 1

E1_CON2: Variable de confianza 2

E1_CON3: Variable de confianza 3

E1_CON4: Variable de confianza 4

E1_CON5: Variable de confianza 5

E1_INT1: Variable de intención 1

E1_INT2: Variable de intención 2

E1_INT3: Variable de intención 3

E1_INT4: Variable de intención 4

E1_INT5: Variable de intención 5 